\title{
Gauge dependence of effective gravitational field
}

\author{
Kirill A. Kazakov* and Petr I. Pronin ${ }^{\dagger}$ \\ June 12, 2018

\begin{abstract}
Moscow State University, Physics Faculty, Department of Theoretical Physics. 117234, Moscow, Russian Federation
\end{abstract}

\begin{abstract}
The problem of gauge independent definition of effective gravitational field is considered from the point of view of the process of measurement. Under assumption that dynamics of the measuring apparatus can be described by the ordinary classical action, effective Slavnov identities for the generating functionals of Green functions corresponding to a system of arbitrary gravitational field measured by means of scalar particles are obtained. With the help of these identities, the total gauge dependence of the non-local part of the one-loop effective apparatus action, describing the long-range quantum corrections, is calculated. The value of effective gravitational field inferred from the effective apparatus action is found to be gauge-dependent. A probable explanation of this result, referring to a peculiarity of the gravitational interaction, is given.
\end{abstract}

PACS 04.60.Ds, 11.10.Lm

\section{Introduction}

Quantization of fields, like any other quantization procedure, contains as one of its inalienable traits an ambiguity in the choice of a set of fundamental variables in terms of which transition from deterministic classical theory to statistical quantum theory is performed. In quantum mechanics this constitutes most of what is called the operatorordering problem. In the quantum theory of fields this appears as the problem of dependence of observables on the choice of field parametrization or, in gauge theories, on the choice of gauge-fixing conditions. Nowhere it is better illustrated than in calculation of the effective fields, i.e. fields incorporating vacuum polarization effects induced by a given classical solutions. Due to special structure of Lagrangians of quantum electrodynamics and Yang-Mills theories, gauge dependence of the corresponding effective fields occurs only beginning with the two-loop approximation of the perturbation theory. Instead, in quantum gravity, dependence of the effective gravitational field on the gauge (and parametrization) is fully displayed already at the one-loop level't.

\footnotetext{
*Email address: kirill@theor.phys.msu.su

$\dagger$ Email address: petr@theor.phys.msu.su

${ }^{1}$ Explicit calculations of the one-loop divergences of the effective action for Einstein gravity and $R^{2}$-gravity in arbitrary gauge and parametrization can be found in [3].
} 
Most generally, the problem under consideration can be stated as the problem of gauge and parametrization dependence of the effective action. The latter can be defined either as the sum over all one-particle-irreducible diagrams [1], or as the Legendre transform of the logarithm of the generating functional of Green functions with respect to the sources [2]. The latter definition brings to light the remarkable analogy between classical equations of motion and the quantum equations describing dynamics of the mean fields. It suggests natural interpretation of the effective action as the quantum substitute for its classical counterpart. However, the above-mentioned problem lacks direct physical application of this analogy.

On the other hand, as we pointed out at the beginning, this "drawback" is an obligatory consequence of the change in the interpretative framework - non-invariance with respect to transitions between different sets of fundamental variables, with the help of which averaging procedures are established, is in the very statistical nature of quantum theory. It disappears only in the classical limit. Although this fact is quite obvious, it should be emphasized that one does not even have to try to prove it, since it is a direct consequence of Bohr's correspondence principle which underlies quantum theory itself. At this point, we would like to recall that classical conceptions play crucial role in another important notion of quantum theory - the process of measurement. As emphasized in $\mathbb{4}$, the very idea of acquisition of some definite reading by a measuring device is essentially classical. In the light of the gauge dependence problem eventual classical nature of any process of measurement becomes particularly important. Namely, it raises the question of whether this problem is just a matter of the formalism of effective action itself, or indicates the necessity to include measuring apparatus into the mathematical description of quantum phenomena explicitly, so as to make it possible to reformulate the theory of effective quantities in terms characterizing motion of the apparatus. If the former is true, then we are left with the S-matrix approach as the only reliable, though very restrictive, means of deriving physical predictions, while the opposite would mean that we get the most general quantum description at our disposall'?

The crucial role of the process of measurement in approaching to the problem of gauge dependence of the effective fields was first put forward by Dalvit and Mazzitelli [5], who showed, in the case of quantum gravity, that the equations of motion (geodesic equation) of a test particle in the weak static effective gravitational field of a point mass, calculated in the one-loop approximation up to leading logarithms, are independent of the choice of linear gauge fixing the general coordinate invariance. The essential thing here is that while the quantum interaction of the test particle with the gravitational field is negligible in evaluation of the total effective field, it is not when the equations of the test particle motion are being determined. It turns out that in the latter case the gauge-dependent contribution originating from the graviton-test particle interaction just cancels that corresponding to the ordinary gauge dependence of the mean gravitational field. Gauge independence of the equations of motion of the classical apparatus allows one to define the gauge independent effective gauge field as the field that enters these equations and couples to the measuring device in the classical fashion.

This important result raises naturally the following questions: first of all, is the

\footnotetext{
${ }^{2}$ This alternative, of course, is exhaustive only as far as the problem of gauge dependence is concerned. An eventual solution to this problem may well turn out to be unsatisfactory from other points of view.
} 
aforesaid cancellation just a lucky accident conditioned by the model chosen and the approximations made in [5], or represents a general property of gauge interactions? Second, if the latter is true, what is the formal mathematical reason underlying the above-mentioned gauge dependence cancellation? That this cancellation is not a mere chance in power-counting-renormalizable theories at least in the low-energy limit is shown quite generally in [6]. There, the use has been made of the ordinary BecchiRouet-Stora-Tyutin (BRST) symmetry [7] of the Faddeev-Popov action [8], which is not, however, a quantum symmetry in the present case, since it includes transformations of the classical matter describing the measuring device. This symmetry is expressed by Slavnov-type identities for Green functions, obtained in [6] and called there effective Slavnov identities. With the help of the renormalization equation following from these identities, gauge independence of the effective equations of device motion is proved in the low-energy limit up to leading logarithms.

The purpose of the present paper is to continue investigation of these matters in the case of quantum gravity. First of all, it is important to consider the role of the measuring apparatus from the field-theoretical point of view, i.e., when the apparatus is described by some classical fields dynamics of which can be determined by the limiting procedure of transition from the quantum to the classical field theory. Second, it is desirable to extend the whole analysis to arbitrary space-time configurations. Significance of the effective Slavnov identities is that they allow one to put the problem of gauge dependence, understood in the sense outlined above, in the most adequate and technically convenient way. Namely, they permit to escape the necessity of explicit evaluation of the mean fields, which requires solving the corresponding wave equations and subsequent substitution of the results into the effective equations of the apparatus motion, in order to verify the gauge dependence cancellation, - the way followed in [5]. Indeed, as shown in [6], the problem of calculation of the total gauge dependence of the effective device action reduces to evaluation of the gauge dependence of connected Green functions containing vertices of the gauge field-device interaction. It is this simplification that allows us to investigate the problem under consideration in arbitrary gravitational fields. This is the subject of the present article. We take as an example the quantized gravitational field measured by a classical scalar field. As in [0], the measuring apparatus is considered as testing (i.e. neglecting its contribution to the total effective gravitational field), so the results are trivially extended to the case when the apparatus is described by an arbitrary number of scalar particles possessing, in particular, internal symmetries (e.g., the pions), the only condition being one and the same structure of interaction with the gravitational field for all particles.

A very important peculiarity of the gravitational interaction must be mentioned here. As was noted in [9], the notion of classical matter looses its usual meaning in the case of gravity, simply because the strength of the gravitational interaction is proportional to the particle mass. As a result, the relative quantum corrections to the equations of motion of the particle do not disappear in the limit $m \rightarrow \infty$. In particular, the whole calculation of the gauge dependence of the effective apparatus action is divided into two large parts: evaluation of the total (in the sense of [5]) gauge dependence of the scalar field effective action when the quantum propagation of the scalar field is neglected (i.e., when its initial action is taken in the usual classical form), and calculation of the gauge dependence of the off-mass-shell scalar field form factors in the limit $m \rightarrow \infty$.

The first part of this program is carried out in this paper. 
Our paper is organized as follows. We begin in Sec. 2 with a more detailed formulation of the gauge dependence problem as well as of the approach to it, adopted in this paper. The effective Slavnov identities for the generating functionals of Green functions, introduced in Sec. 3, are derived in Sec. 4. In essential, this derivation reproduces that given in [6]. In Sec. 5, these identities are used in evaluation of the logarithmic contribution to the $\xi$-dependent part of the generating functional of connected Green functions, $\xi$ being the gauge parameter weighting Lorentz-type gauge condition $a \partial^{\mu} h_{\mu \nu}+b \eta^{\alpha \beta} \partial_{\nu} h_{\alpha \beta}=0$. We find that, unlike the case of the point-like measuring apparatus considered in [5], this contribution is not zero. The results obtained are discussed in Sec. 6.

We use the highly condensed notations of DeWitt [10] throughout this paper. Also left derivatives with respect to anticommuting variables are used. The dimensional regularization of all divergent quantities is supposed.

\section{Preliminaries}

Before we proceed to calculations, we would like to give a somewhat more detailed account of the main aspects of the gauge dependence problem, briefly mentioned in the Introduction.

\subsection{The origin of the problem}

First of all, we would like to point out the close connection of the gauge dependence problem with the analogous problem of dependence of the effective action on the choice of field parametrization. Roughly speaking, imposition of the gauge conditions in a gauge theory, being equivalent to picking a subset out of the total set of variables describing the theory, includes, in particular, freedom to perform arbitrary substitutions among the variables of the subset. The parametrization dependence problem is just the reflection of this freedom possessed by any gauge as well as non-gauge field theory. As a matter of fact, any like the above reasoning has restricted validity in field theory because of the fundamental locality requirements. Nevertheless, it is a general result of the Batalin-Vilkovisky method [12] that the behavior of the effective action under variations of the gauge conditions is essentially the same as under arbitrary changes of parametrization - both are represented by anticanonical transformations (in the sense

of the Batalin-Vilkovisky antibracket). This result is established in full generality in 113 and, furthermore, is valid in renormalized as well as unrenormalized theory. In view of this fact, one can speak about either gauge or parametrization dependence of the effective action.

Let us now turn to the problem itself. As we have already mentioned above, its origin is in the inevitable ambiguity in the choice of a set of fundamental variables in terms of which the quantization procedure is carried out. Consider a simple example. Let the system be described by an action $S(\varphi)$ which is a function of a single scalar field $\varphi(x)$ and suppose that the functional integral measure can be chosen simply as the product of $d \varphi(x)$. Then the mean field $\varphi(y)$ is

$$
\langle\varphi(y)\rangle_{\varphi}=\int \prod_{x} d \varphi(x) \varphi(y) \exp \{i S(\varphi)\}
$$


The subscript $\varphi$ indicates that the role of fundamental dynamical variable is played here by the field $\varphi$ itself. Nothing prevents us, however, from taking as fundamental any other field $\varphi^{*}=f[\varphi], f[\varphi]$ being an arbitrary (local) non-degenerate function, in which case Eq. (1) is replaced by

$$
\begin{aligned}
\left\langle\varphi^{*}(y)\right\rangle_{\varphi^{*}} & =\int \prod_{x} d \varphi^{*}(x) \varphi^{*}(y) \exp \left\{i S^{*}\left(\varphi^{*}\right)\right\}, \\
S^{*}\left(\varphi^{*}\right) & \equiv S\left(f^{-1}\left[\varphi^{*}\right]\right) .
\end{aligned}
$$

Employing dimensional regularization, so that $\delta(0)$-type expressions are set equal to zero, we rewrite Eq. (21) as

$$
\left\langle\varphi^{*}(y)\right\rangle_{\varphi^{*}}=\int \prod_{x} d \varphi(x) f[\varphi(y)] \exp \{i S(\varphi)\}=\langle f[\varphi(y)]\rangle_{\varphi} .
$$

Since there is no reason to prefer one way of quantization to the other, in particular, one definition of the mean field to the other, one can try at least to compare the two. Obviously, the only way to do this is to use the relation $\varphi^{*}=f[\varphi]$. Consider, e.g., the $\varphi$ picture. On the one hand, as it follows from Eq. (11), the mean field is equal to $\langle\varphi(y)\rangle_{\varphi}$. On the other hand, one is equally right to take for it the value $f^{-1}\left[\left\langle\varphi^{*}(y)\right\rangle_{\varphi^{*}}\right]$. Thus, taking into account Eq. (3), we conclude that the change $\varphi^{*}=f[\varphi]$ of fundamental variables leads to the following change in the value of the mean field

$$
\langle\varphi(y)\rangle_{\varphi} \rightarrow f^{-1}\left[\langle f[\varphi(y)]\rangle_{\varphi}\right] .
$$

Conversely, in the $\varphi^{*}$ picture, one arrives at the rule

$$
\left\langle\varphi^{*}(y)\right\rangle_{\varphi^{*}} \rightarrow f\left[\left\langle f^{-1}\left[\varphi^{*}(y)\right]\right\rangle_{\varphi^{*}}\right] .
$$

Assumptions concerning the regularization scheme and the function $f[\varphi]$, made above, play no role: the same transformation rule (बi) applies in the general case, as it follows from the results of [14]. Obviously, ambiguity expressed by this rule is an indispensable consequence of statistical nature of quantum field theory together with complete equivalence of various pictures (i.e., formulations in various sets of variables) at the classical level. We would like to emphasize in this connection that the very use of the relation $\varphi^{*}=f[\varphi]$ in comparison of the two quantum pictures above is by itself essentially classical. Yet this deprives the transformations (蛋) of any significance in the quantum domain, whereas at the classical level these turn, of course, into a trivial identity. Nevertheless, requirement of invariance with respect to the transformations (4i) is taken in [15] as the starting point in construction of a modification of the ordinary effective action, aimed at derivation of physically sensible predictions. As it follows from what we just said, apart from the question of whether the action proposed in [15] is actually gauge-independent, such an object would have nothing to do with the genuine problem of gauge dependence. Instead, one is prompted by the above discussion to seek the way out of this "difficulty" in reformulation of the theory in classical terms. This is exactly what is done in [5]. Effective gravitational field is defined there through the effective equations of motion of a classical test particle, namely, as the field that takes place of the classical gravitational field in the ordinary geodesic equation. Obviously, this is the only definition relevant to the state of affairs in the light of the process of measurement. Indeed, in any case it is motion of specific classical apparatus measuring 
the field at any given space-time interval that is only observed. Thus, it is value of the field entering classical equations of motion of the apparatus that is only important.

Following [5], we consider the apparatus as testing, i.e., as an infinitely small disturbance of the gravitational field it measures. Let us now show how this technical assumption allows one to simplify the calculations to be performed below.

\subsection{The role of simple connectedness}

Since we are interested mainly in answering the question of whether the effective field, defined in the sense of Dalvit and Mazzitelli, is actually gauge independent, rather than in its specific value, especially in the case of quantum gravity, we can simplify evaluation of the total gauge-dependent part of the effective device action as follows. As is explained in the Introduction, this part is the sum of two different contributions. The first is the ordinary explicit gauge dependence of the effective device action. The second stems from its implicit gauge dependence through the mean gauge field. It is exactly this dependence of the mean gravitational field that lacks its physical interpretation. Let the measuring apparatus be described by a set of classical fields, denoted as $\phi$. Then, if $\Gamma_{\phi}$ stands for the $\phi$-dependent part of the generating functional of one-particleirreducible Green functions, $\Gamma$, we have for the full variation of the effective device action under a small change $\delta \xi$ of a gauge parameter $\xi$ :

$$
\delta \Gamma_{\phi}(h, \phi, \xi)=\left.\frac{\partial \Gamma_{\phi}}{\partial \xi}\right|_{h, \phi} \delta \xi+\left.\frac{\delta \Gamma_{\phi}}{\delta h_{\mu \nu}} \frac{\partial h_{\mu \nu}}{\partial \xi}\right|_{T, \phi} \delta \xi \equiv \frac{d \Gamma_{\phi}}{d \xi} \delta \xi,
$$

where $T \equiv\left\{T^{\mu \nu}\right\}$ is the source for the gravitational field, and $h \equiv\left\{h_{\mu \nu}\right\}$ is the mean deviation of the metric from Minkowskian.

Now note, that if the quantity $W_{\phi}$ is defined by analogy with $\Gamma_{\phi}$, i.e., as the $\phi$ dependent part of the generating functional of connected Green functions, $W$, then

$$
\Gamma_{\phi}(h, \phi, \xi)=\left.W_{\phi}(T, \phi, \xi)\right|_{T \rightarrow T(h, \xi)},
$$

since the device contribution is infinitesimal.

Comparing Eqs. (6) and ([7), we arrive at the following important relation

$$
\left.\frac{d \Gamma_{\phi}(h, \phi, \xi)}{d \xi}\right|_{h \rightarrow h(T, \xi)}=\frac{\partial W_{\phi}(T, \phi, \xi)}{\partial \xi} .
$$

Due to this result, transition to the strongly connected diagrams in the effective Slavnov identities derived below becomes unnecessary.

\section{The quantum action and the generating function- als}

The method we approach the gauge dependence problem allows consideration of arbitrary space-time configurations. The simplest way to set up any desired is to choose properly the standard source term normally introduced into the generating functional of Green functions. Thus, the role of the field $T^{\mu \nu}$ introduced already in Eqs. (6-8) will be not only to serve as the variable of the Legendre transformation, but also to provide 
a convenient substitute for realistic matter sources. The only trace of the latter is the "conservation law"

$$
\nabla_{\mu} T^{\mu \nu}=0
$$

where the covariant derivatives are defined with respect to the metric $h^{0}$ satisfying

$$
\frac{\delta\left(S+S_{g f}\right)}{\delta h_{\mu \nu}}=-T^{\mu \nu} .
$$

$S$ is here the action for the gravitational field"

$$
S=-\frac{1}{k^{2}} \int d^{4} x \sqrt{-g} R
$$

$k$ being the gravitational constant

$$
S_{g f}=\frac{1}{2 \xi} \eta^{\alpha \beta} F_{\alpha} F_{\beta}, \quad F_{\alpha}=\partial^{\mu} h_{\mu \alpha}-\frac{1+\beta}{2} \partial_{\alpha} h, \quad h \equiv \eta^{\mu \nu} h_{\mu \nu}
$$

$F$ is the most general covariant gauge condition linear in $h$. Finally, we assume that the measuring device can be described by a single scalar field $\phi$, its action being

$$
S_{\phi}=\frac{1}{2} \int d^{4} x \sqrt{-g}\left(g^{\mu \nu} \partial_{\mu} \phi \partial_{\nu} \phi-m^{2} \phi^{2}\right) .
$$

The action $S+S_{\phi}$ is invariant under the following (infinitesimal) gauge transformations?

$$
\begin{aligned}
\delta h_{\mu \nu} & =\xi^{\alpha} \partial_{\alpha} h_{\mu \nu}+\left(\eta_{\mu \alpha}+h_{\mu \alpha}\right) \partial_{\nu} \xi^{\alpha}+\left(\eta_{\nu \alpha}+h_{\nu \alpha}\right) \partial_{\mu} \xi^{\alpha} \equiv D_{\mu \nu}^{\alpha}(h) \xi_{\alpha} \\
\delta \phi & =\xi^{\alpha} \partial_{\alpha} \phi \equiv \tilde{D}^{\alpha}(\phi) \xi_{\alpha}
\end{aligned}
$$

where $\xi^{\alpha}$ are the (infinitesimal) gauge functions. The generators $D, \tilde{D}$ span the closed algebra

$$
\begin{aligned}
& D_{\mu \nu}^{\alpha, \sigma \lambda} D_{\sigma \lambda}^{\beta}-D_{\mu \nu}^{\beta, \sigma \lambda} D_{\sigma \lambda}^{\alpha}=f^{\alpha \beta}{ }_{\gamma} D_{\mu \nu}^{\gamma}, \\
& \tilde{D}_{1}^{\alpha} \tilde{D}^{\beta}-\tilde{D}_{1}^{\beta} \tilde{D}^{\alpha}=f_{\gamma}^{\alpha \beta} \tilde{D}^{\gamma},
\end{aligned}
$$

where the "structure constants" $f_{\gamma}^{\alpha \beta}$ are defined by

$$
f_{\gamma}^{\alpha \beta} \xi_{\alpha} \eta_{\beta}=\xi_{\alpha} \partial^{\alpha} \eta_{\gamma}-\eta_{\alpha} \partial^{\alpha} \xi_{\gamma}
$$

Next, introducing Faddeev-Popov ghost fields $C_{\alpha}, \bar{C}^{\alpha}$ we write Faddeev-Popov quantum action

$$
S_{f p}=S+S_{\phi}+S_{g f}+\bar{C}^{\beta} F_{\beta}^{, \mu \nu} D_{\mu \nu}^{\alpha} C_{\alpha} .
$$

\footnotetext{
${ }^{3}$ Our notation is $R_{\mu \nu} \equiv R_{\mu \alpha \nu}^{\alpha}=\partial_{\alpha} \Gamma_{\mu \nu}^{\alpha}-\ldots, R \equiv R_{\mu \nu} g^{\mu \nu}, g \equiv \operatorname{det}_{\mu \nu}, g_{\mu \nu}=\operatorname{sgn}(+,-,-,-)$. Dynamical variables of the gravitational field $h_{\mu \nu}=g_{\mu \nu}-\eta_{\mu \nu}, \eta_{\mu \nu}=\operatorname{diag}\{+1,-1,-1,-1\}$.

${ }^{4}$ We choose units in which $c=\hbar=k=1$ from now on.

${ }^{5}$ Indices of the functions $F, \xi$, as well as of the ghost fields below, are raised and lowered, if convenient, with the help of Minkowski metric $\eta_{\mu \nu}$.
} 
$S_{f p}$ is still invariant under the following BRST transformations [7]

$$
\begin{aligned}
\delta_{b r s t} h_{\mu \nu} & =D_{\mu \nu}^{\alpha}(h) C_{\alpha} \lambda, \\
\delta_{b r s t} C_{\gamma} & =-\frac{1}{2} f^{\alpha \beta}{ }_{\gamma} C_{\alpha} C_{\beta} \lambda, \\
\delta_{b r s t} \bar{C}^{\alpha} & =\frac{1}{\xi} F^{\alpha} \lambda, \\
\delta_{b r s t} \phi & =\tilde{D}^{\alpha}(\phi) C_{\alpha} \lambda,
\end{aligned}
$$

$\lambda$ being a constant anticommuting parameter.

BRST transformation rule for the $\phi$-field is here separated from the rest to emphasize the special role played by the measuring device in the present formalism. On the one hand, Eqs. (18), (19) span the usual BRST transformation of the quantum action (17). On the other hand, in derivation of the effective Slavnov identities below, the $\phi$-field being classical does not take a part in the quantum BRST transformation, i.e., transformation of the path integral measure in the generating functional of Green functions

$$
Z[T, \bar{\beta}, \beta, K, \tilde{K}, L]=\int d h d C d \bar{C} \exp \left\{i\left(\Sigma+\bar{\beta}^{\alpha} C_{\alpha}+\bar{C}^{\alpha} \beta_{\alpha}+T^{\mu \nu} h_{\mu \nu}\right)\right\},
$$

where

$$
\Sigma=S_{f p}+K^{\mu \nu} D_{\mu \nu}^{\alpha} C_{\alpha}+\tilde{K} \tilde{D}^{\alpha} C_{\alpha}+\frac{1}{2} L^{\gamma} f_{\gamma}^{\alpha \beta} C_{\alpha} C_{\beta}
$$

$K^{\mu \nu}(x), \tilde{K}(x)$ (anticommuting), $L^{\alpha}(x)$ (commuting) being the BRST transformation sources [16].

Before we continue, we would like to make some notes on the form of the generating functional (20). It is because of its classical nature the field $\phi$ is absent in the functional integral measure in Eq. (20). The quantum propagation of this field is thereby neglected. Furthermore, the same classical nature allows $\phi$-field to be considered as a c-function. In terms of the creation $\hat{a}^{\dagger}$ and annihilation $\hat{a}$ operators this is expressed as the approximate commutativity

$$
\hat{a} \hat{a}^{\dagger}-\hat{a}^{\dagger} \hat{a} \approx 0
$$

justified if the occupation numbers of the quantum states involved are large compared with the unity. It is worth to recall in this connection that in quantum field theory the classical requirements can be applied only to a finite, although otherwise arbitrary, region of field spectrum. In particular, the above condition on the value of the occupation numbers inevitably becomes meaningless when applied to all energies.

We see, therefore, that the above form of the generating functional is an immediate consequence of Bohr's correspondence principle underlying the whole quantum theory.

It is to be mentioned, however, that it is in the case of the gravitational interaction where the usual procedure of transition to the classical limit does not work. Namely, as we mentioned in the Introduction, it is senseless to increase the apparatus mass in

\footnotetext{
${ }^{6}$ For brevity, the product symbol, as well as tensor indices of the fields $h_{\mu \nu}, C_{\alpha}, \bar{C}^{\alpha}$, is omitted in the path integral measure.
} 
order to suppress its quantum contribution, since the same mass multiplies vertices of the graviton-apparatus interaction. In short, there is no such thing as the classical source for gravity [9]. In this paper, we follow [5], and put the device action into the classical form (13) "by hands".

As we mentioned in Sec. 2.1, the parametrization dependence problem is in fact a part of the more general problem of gauge dependence. Below we consider the latter case as the more familiar. To illustrate the essence of our approach as well as of the main result it is sufficient to consider the most important kind of the gauge dependence, namely, dependence on the weighting parameter $\xi$. To accomplish this, we modify the quantum action adding the term

$$
Y F_{\alpha} \bar{C}^{\alpha}
$$

$Y$ being a constant anticommuting parameter [17]. Thus we write the generating functional of Green functions as

$$
\begin{aligned}
& Z[T, \bar{\beta}, \beta, K, \tilde{K}, L, Y]=\int d h d C d \bar{C} \exp \{i(\Sigma \\
& \left.\left.+Y F_{\alpha} \bar{C}^{\alpha}+\bar{\beta}^{\alpha} C_{\alpha}+\bar{C}^{\alpha} \beta_{\alpha}+T^{\mu \nu} h_{\mu \nu}\right)\right\} .
\end{aligned}
$$

To complete our definitions, we introduce the generating functional of connected Green functions

$$
\tilde{W}[T, \bar{\beta}, \beta, K, \tilde{K}, L, Y]=-i \ln Z[T, \bar{\beta}, \beta, K, \tilde{K}, L, Y]
$$

and then define the effective action $\tilde{\Gamma}$ in the usual way as the Legendre transform of $\tilde{W}$ with respect to the mean fields

$$
\begin{gathered}
h_{\mu \nu}=\frac{\delta \tilde{W}}{\delta T^{\mu \nu}}, \\
C_{\alpha}=\frac{\delta \tilde{W}}{\delta \bar{\beta}^{\alpha}}, \\
\bar{C}^{\alpha}=-\frac{\delta \tilde{W}}{\delta \beta_{\alpha}},
\end{gathered}
$$

(denoted by the same symbols as the corresponding field operators):

$$
\begin{aligned}
& \tilde{\Gamma}[h, C, \bar{C}, K, \tilde{K}, L, Y]=\tilde{W}[T, \bar{\beta}, \beta, K, \tilde{K}, L, Y] \\
& -\bar{\beta}^{\alpha} C_{\alpha}-\bar{C}^{\alpha} \beta_{\alpha}-T^{\mu \nu} h_{\mu \nu},
\end{aligned}
$$

In the standard interpretation, the reciprocal to equations (23),

$$
\frac{\delta \Gamma}{\delta h_{\mu \nu}}=-T^{\mu \nu}, \quad \Gamma[h] \equiv \tilde{\Gamma}[h, 0,0, \ldots]
$$

are the effective equations of motion for the full quantum corrected field $h_{\mu \nu}$. 


\section{The effective Slavnov identities}

In terms of Green functions, the symmetry with respect to the transformations (14) is expressed by Slavnov-type identities, to derive which we perform a BRST shift (18) of integration variables in the path integral (21). Unlike the usual case, however, the modified quantum action $\Sigma$ is not invariant under this operation, since, as we have mentioned above, the classical field $\phi$ does not take a part in it. Therefore, we obtain the following identity

$$
\begin{aligned}
& 0=\int d h d C d \bar{C}\left[i \frac{\delta S_{\phi}}{\delta h_{\mu \nu}} D_{\mu \nu}^{\alpha} C_{\alpha}+i Y \bar{C}^{\alpha} F_{\alpha}^{, \mu \nu} D_{\mu \nu}^{\beta} C_{\beta}+i \frac{Y}{\xi} F_{\alpha}^{2}-\frac{i}{2} \tilde{K} \tilde{D}^{\gamma} f_{\gamma}^{\alpha \beta} C_{\alpha} C_{\beta}\right. \\
& \left.+T^{\mu \nu} \frac{\delta}{\delta K^{\mu \nu}}-\bar{\beta}^{\alpha} \frac{\delta}{\delta L^{\alpha}}-i \beta_{\alpha} \frac{F^{\alpha}}{\xi}\right] \exp \left\{i\left(\Sigma+Y F_{\alpha} \bar{C}^{\alpha}+\bar{\beta}^{\alpha} C_{\alpha}+\bar{C}^{\alpha} \beta_{\alpha}+T^{\mu \nu} h_{\mu \nu}\right)\right\} .
\end{aligned}
$$

Since $S_{\phi}$ is itself invariant under BRST-transformations (18), (19),

$$
\frac{\delta S_{\phi}}{\delta h_{\mu \nu}} D_{\mu \nu}^{\alpha} C_{\alpha}=-\frac{\delta S_{\phi}}{\delta \phi} \tilde{D}^{\alpha} C_{\alpha}
$$

Using this identity, we rewrite the first term in the square brackets on the left of Eq. (27) as follows

$$
\begin{aligned}
& \int d h d C d \bar{C} \frac{\delta S_{\phi}}{\delta h_{\mu \nu}} D_{\mu \nu}^{\alpha} C_{\alpha} \exp \{\cdots\}=-\int d h d C d \bar{C} \frac{\delta S_{\phi}}{\delta \phi} \tilde{D}^{\alpha} C_{\alpha} \exp \{\cdots\} \\
& =-\frac{1}{i} \frac{\delta}{\delta \phi} \int d h d C d \bar{C} \tilde{D}^{\alpha} C_{\alpha} \exp \{\cdots\}+\int d h d C d \bar{C} \tilde{D}^{\alpha} C_{\alpha} \tilde{K}^{\delta} \frac{\delta \tilde{D}^{\beta}}{\delta \phi} C_{\beta} \exp \{\cdots\} \\
& =\frac{\delta^{2} Z}{\delta \phi \delta \tilde{K}}+\int d h d C d \bar{C} \tilde{K} \tilde{D}^{\gamma} \frac{1}{2} f_{\gamma}^{\alpha \beta} C_{\alpha} C_{\beta} \exp \{\cdots\},
\end{aligned}
$$

where the second of Eqs. (15), and the locality of generator $\tilde{D}(\phi)$ together with the property $\delta(0)=0$ were taken into account.

Next, the second term in the square brackets in Eq. (27) can be transformed with the help of the quantum ghost equation of motion, obtained by performing a shift $\bar{C} \rightarrow \bar{C}+\delta \bar{C}$ of integration variables in the functional integral (20):

$$
\begin{aligned}
& \int d h d C d \bar{C}\left[F_{\gamma}^{, \mu \nu} D_{\mu \nu}^{\alpha} C_{\alpha}-Y F_{\gamma}+\beta_{\gamma}\right] \\
& \times \exp \left\{i\left(\Sigma+Y F_{\alpha} \bar{C}^{\alpha}+\bar{\beta}^{\alpha} C_{\alpha}+\bar{C}^{\alpha} \beta_{\alpha}+T^{\mu \nu} h_{\mu \nu}\right)\right\}=0,
\end{aligned}
$$

from which it follows that

$$
Y \int d h d C d \bar{C}\left[i \bar{C}^{\gamma} F_{\gamma}^{, \mu \nu} D_{\mu \nu}^{\alpha} C_{\alpha}+\beta_{\gamma} \frac{\delta}{\delta \beta_{\gamma}}\right] \exp \{\cdots\}=0,
$$

where the use of the property $Y^{2}=0$ has been made, and the expression $\delta \beta_{\gamma} / \delta \beta_{\gamma} \sim \delta(0)$ is again omitted. Putting this all together, we rewrite Eq. (27)

$$
\left(T^{\mu \nu} \frac{\delta}{\delta K^{\mu \nu}}-\bar{\beta}^{\alpha} \frac{\delta}{\delta L^{\alpha}}-\frac{1}{\xi} \beta_{\alpha} F^{\alpha, \mu \nu} \frac{\delta}{\delta T^{\mu \nu}}+i \frac{\delta^{2}}{\delta \phi \delta \tilde{K}}-Y \beta_{\gamma} \frac{\delta}{\delta \beta_{\gamma}}-2 Y \xi \frac{\partial}{\partial \xi}\right) Z=0 .
$$


This is the effective Slavnov identity for the generating functional of Green functions we are looking for. In the case $L=\beta=\bar{\beta}=0$ it was obtained in [6]. In terms of the generating functional of connected Green functions, it looks like

$$
T^{\mu \nu} \frac{\delta \tilde{W}}{\delta K^{\mu \nu}}-\bar{\beta}^{\alpha} \frac{\delta \tilde{W}}{\delta L^{\alpha}}-\frac{1}{\xi} \beta_{\alpha} F^{\alpha, \mu \nu} \frac{\delta \tilde{W}}{\delta T^{\mu \nu}}+i \frac{\delta^{2} \tilde{W}}{\delta \phi \delta \tilde{K}}-\frac{\delta \tilde{W}}{\delta \phi} \frac{\delta \tilde{W}}{\delta \tilde{K}}-Y \beta_{\gamma} \frac{\delta \tilde{W}}{\delta \beta_{\gamma}}=2 Y \xi \frac{\partial \tilde{W}}{\partial \xi} .
$$

Appearance of the second derivatives in Eqs. (30), (31) is to be emphasized. Recall that it is presence of higher derivatives (with respect to the sources or mean fields) that makes application of the original formulation of Slavnov identities much less straightforward than of their later reformulation in terms of the sources of BRST transformations, given by Zinn-Justin [16] and used in our derivation above. However, even the latter approach does not prevent appearance of the second derivatives in the effective Slavnov identities. Although the relation (\$) makes this fact unimportant for our consideration, an alternative choice of the quantum action in the generating functional (21) exists which allows one to overcome it, and to obtain the effective Slavnov identities for proper vertices in the general case of finite contribution of the measuring device to the effective gravitational field. This is done in the Appendix.

\section{Gauge dependence of leading low-energy quan- tum corrections}

In this section, we present detailed evaluation of the total $\xi$-dependence of the logarithmic contribution to the effective device action in arbitrary gravitational field. As we have seen, this dependence is given by the functional $\partial W_{\phi} / \partial \xi$, which is conveniently calculated with the help of the effective Slavnov identity (31).

Setting $L=\beta=\bar{\beta}=0$ in Eq. (31) and extracting its $Y$-dependent part, we have

$$
2 \xi \frac{\partial W}{\partial \xi}=-T^{\mu \nu} \frac{\delta \bar{W}}{\delta K^{\mu \nu}}+\frac{\delta W}{\delta \phi} \frac{\delta \bar{W}}{\delta \tilde{K}}-i \frac{\delta^{2} \bar{W}}{\delta \phi \delta \tilde{K}},
$$

where $W, \bar{W}$ are defined by

$$
\tilde{W}=W+Y \bar{W}
$$

and the sources $K^{\mu \nu}, \tilde{K}$ are also set zero after differentiation.

Finally, extraction of the $\phi$-dependent part of Eq. (32) gives

$$
2 \xi \frac{\partial W_{\phi}}{\partial \xi}=-T^{\mu \nu} \frac{\delta \bar{W}_{\phi}}{\delta K^{\mu \nu}}+\frac{\delta W_{\phi}}{\delta \phi} \frac{\delta \bar{W}_{\bar{\phi}}}{\delta \tilde{K}}-i \frac{\delta^{2} \bar{W}_{\phi}}{\delta \phi \delta \tilde{K}}
$$

where $\bar{W}_{\bar{\phi}}$ denotes the $\phi$-independent part of the functional $\bar{W}$. Since the source $\tilde{K}$ is set here equal to zero after differentiation, the notion of $\phi$-dependence retains its meaning given in Sec. 2.2.

Thus, to determine the total $\xi$-dependence of the effective apparatus action one has to evaluate the right hand side of Eq. (33). However, because of zero dimensionality of the gravitational field calculation of infinity of diagrams with arbitrary number of external graviton lines is required. The standard way round this difficulty is the use of the background field method together with an appropriate choice of the gauge 
conditions [10]. This leads to the explicitly gauge invariant effective action [1], which allows one to confine the calculation by the lowest order of the weak field approximation, and then restore the whole gauge invariant contribution on dimensional grounds. It should be emphasized, however, that in our case the very use of this method is under question. Indeed, functional dependence of the gauge conditions on the background field is to be considered here on equal footing with the dependence on the gauge parameters $\xi, \beta$. Therefore, the background field method itself can be used only after the effective apparatus action is shown to be independent of such modification of the gauge conditions, just like as in the $S$-matrix approach the use of this method is justified by the gauge independence of the scattering matrix.

It is worthwhile to note that exactly the background field method was used in [5] in evaluating the quantum corrections to the geodesic equation, as an intermediate step of which one had to solve the effective equations of motion for the (background) gravitational field. It is, however, the main achievement of the method that these equations are gauge invariant [11]. As was emphasized in [5], to solve them one is free to choose any gauge conditions. The solution depends, of course, on this choice, but this is the ordinary harmless gauge dependence encountered in any classical gauge theory, which does not lead to gauge dependence of gauge invariant functionals of this solution. This, however, raises the question which of the two sets of gauge conditions - the one used to fix the gauge freedom of integration variables in the path integral, or the one used to solve the equations of motion - is to be considered as the gauge fixing for the effective gravitational field defined in the sense of Dalvit and Mazzitelli) It seems that, in the background field method, it is the gauge conditions imposed on the background field, which are to be considered also as the gauge-fixing for the effective field, though without detailed proof this is only a probable conjecture.

It should be emphasized, however, that all these are only particularities of the background field method itself. We do not use this method in our approach, in which the gauge freedom of the mean gravitational field is automatically fixed by the conditions imposed on the path integral variables, so the above alternative is never raised. On the other hand, the questions of method are actually irrelevant to the problem under consideration, since the very classical nature of the measuring device implies that its effective action is gauge invariant. Thus, if the lowest order contribution to the gaugedependent part of the effective device action is zero, then the whole contribution must be zero too.

Let us now proceed to evaluation of the right hand side of Eq. (33) in the lowest order of the weak field approximation. As the subsequent calculation shows, the result is linear in the curvature, so the lowest non-vanishing contribution is given by diagrams with one external graviton, pictured in Figs. 1, 2, and 3 representing the first, second, and third term in the right hand side of Eq. (33), respectively. Note that the diagrams of Fig. 3(a,b,c) are obtained from the tree diagrams shown in Fig. 田(a,b,c), respectively, by confluence of pairs of vertices, as shown by the long arrows.

In the first order approximation, Einstein equations read

$$
R^{\mu \nu}-\frac{1}{2} \eta^{\mu \nu} R^{\alpha \beta} \eta_{\alpha \beta}+T^{\mu \nu}=0
$$

\footnotetext{
${ }^{7}$ In [5], the two are simply taken to coincide, putting thereby the question off.

${ }^{8}$ In the case of singular gauge conditions, the gauge fixing for the mean field simply coincides with that for the integration variables. In general, connection between the two is more complicated.
} 


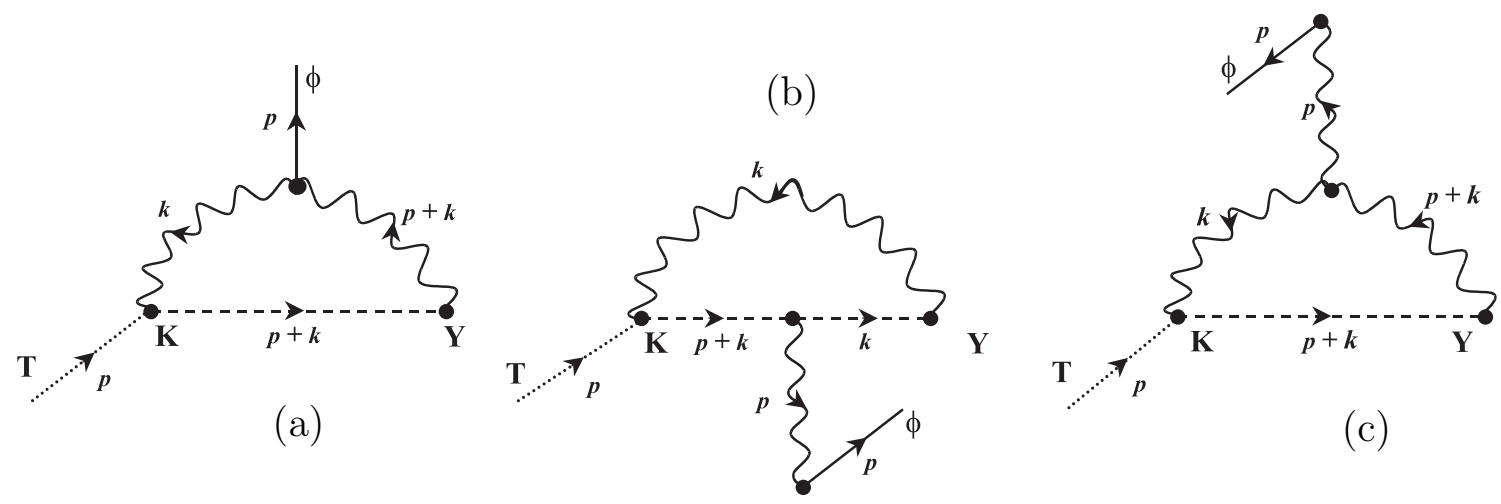

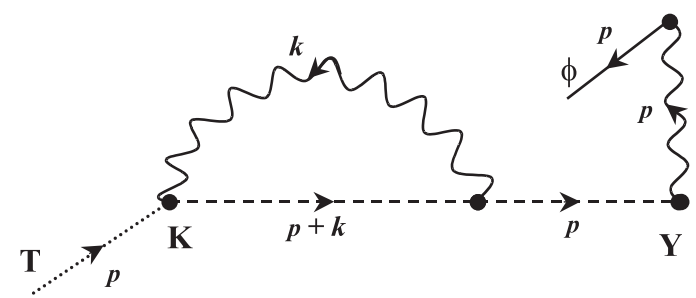

(d)

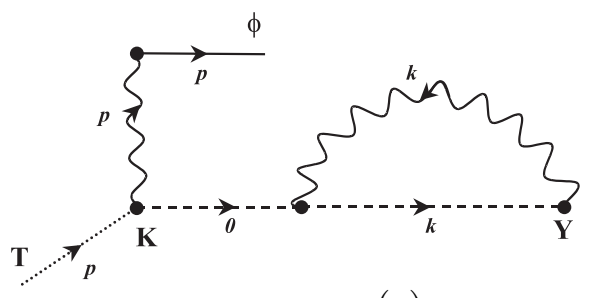

(e)
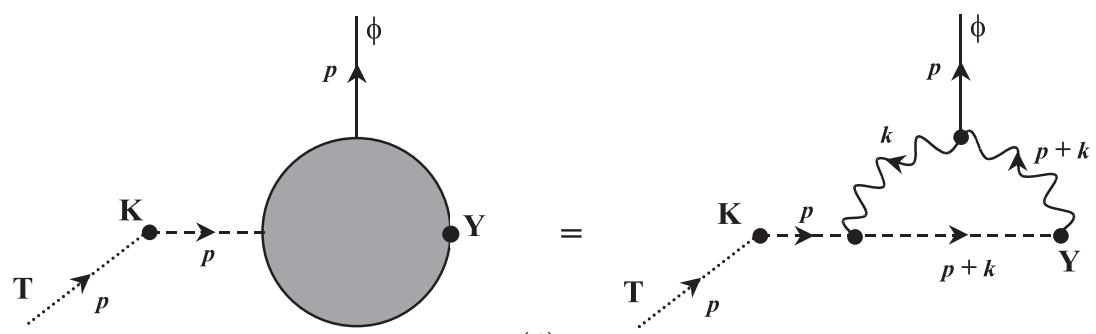

(f)

Figure 1: Diagrams corresponding to the first term in the right hand side of Eq. (33). Wavy lines represent gravitons, dashed lines ghosts, dotted lines the source $T$ for the gravitational field, and solid lines classical field describing the measuring device. Note that the latter denote collectively various functionals of the field $\phi$, encountered in Eq. (33). As explained in the text, diagrams of the type shown in Fig. 1(f) do not contribute, so we do not picture them in detail. 

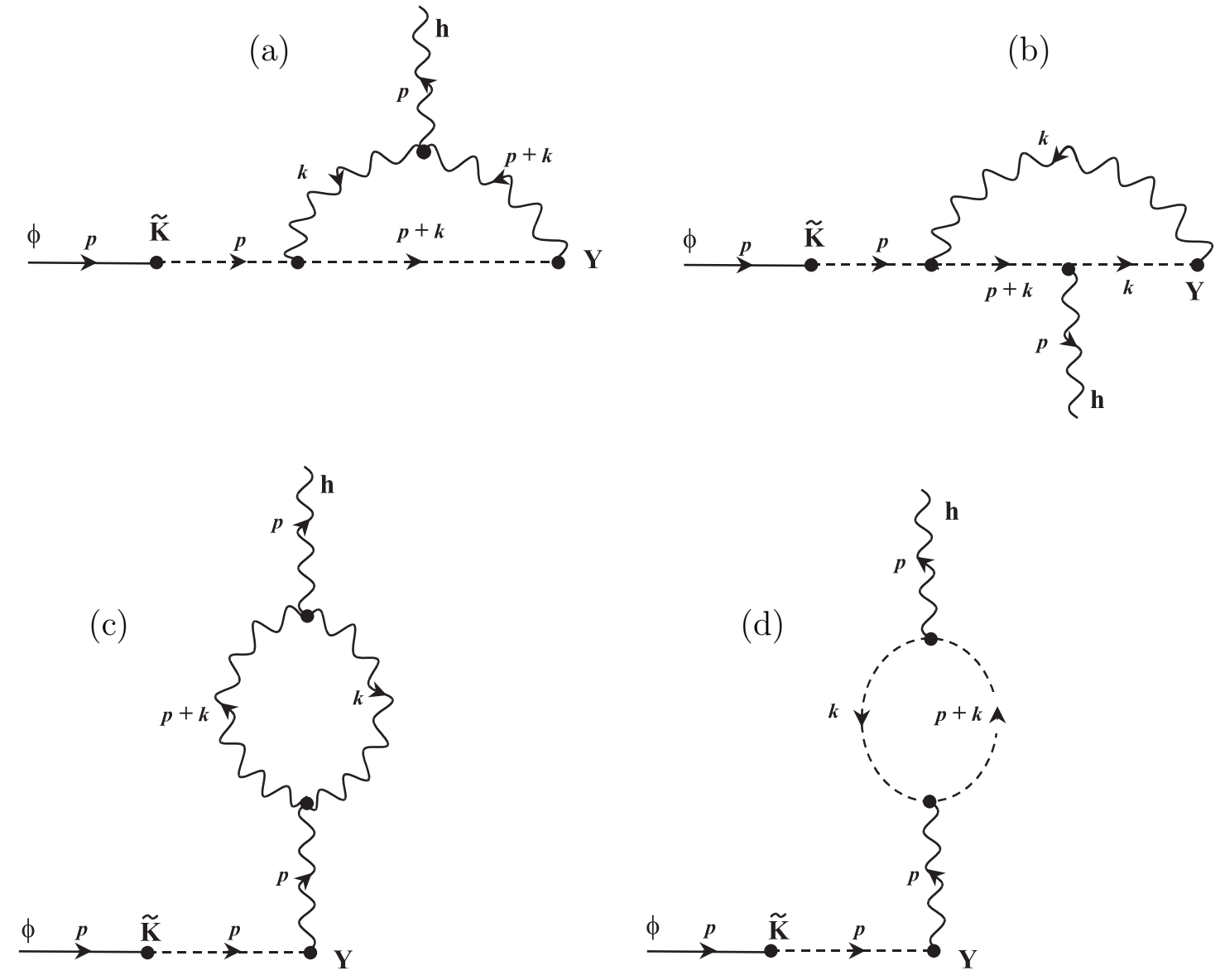

Figure 2: Diagrams corresponding to the second term in the right hand side of Eq. (33).
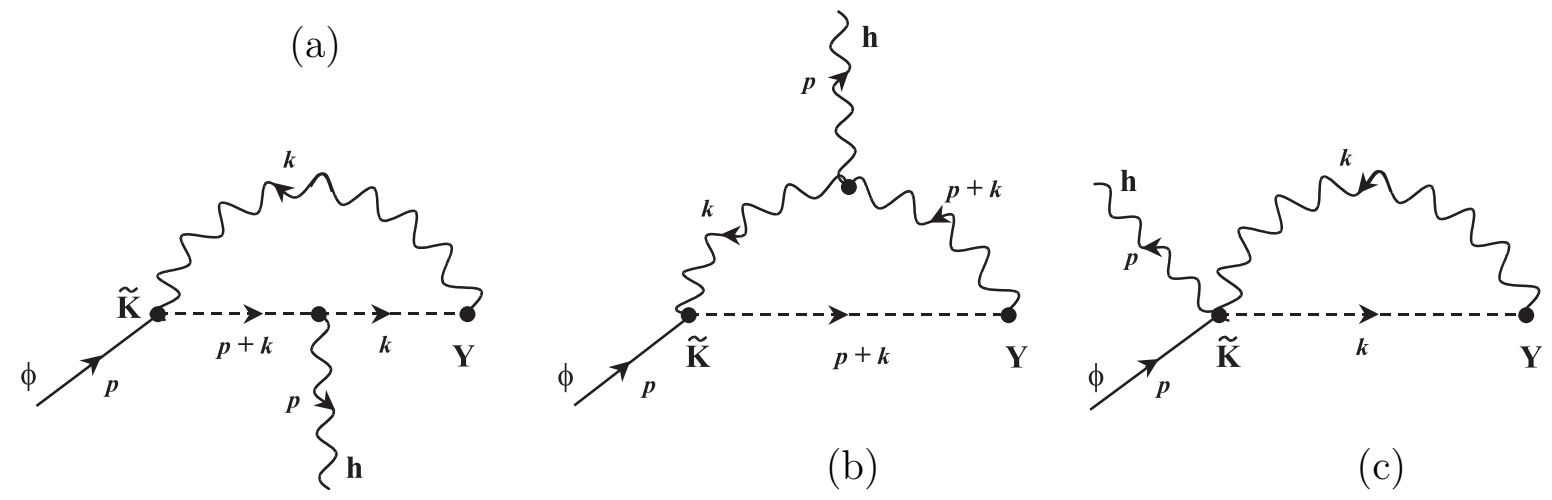

(b)

(c)

Figure 3: Diagrams corresponding to the third term in the right hand side of Eq. (33). They are obtained from tree diagrams as shown in Fig. 4. 


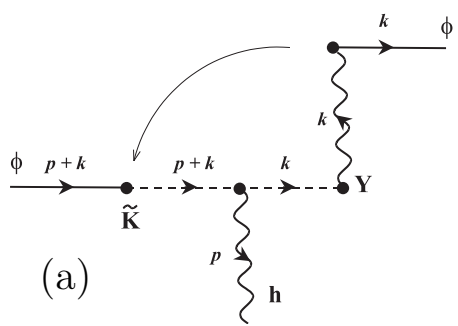

(a)

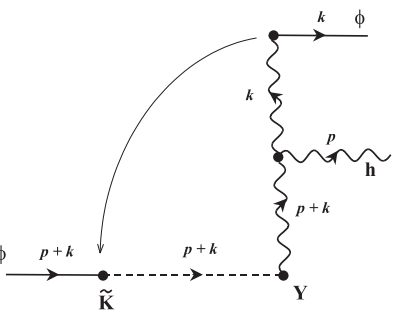

(b)

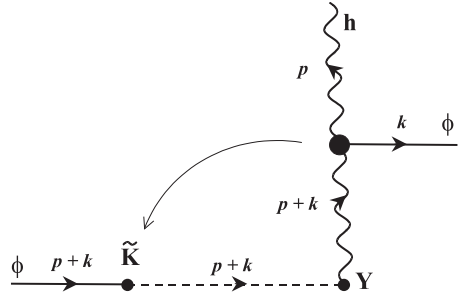

(c)

Figure 4: The tree diagrams from which the one-loop diagrams of Fig. 3 are formed by confluence of vertices, shown by the long arrows.

where

$$
\begin{aligned}
& R_{\mu \nu}=\frac{1}{2}\left(\partial^{\alpha} \partial_{\mu} h_{\alpha \nu}+\partial^{\alpha} \partial_{\nu} h_{\alpha \mu}-\partial^{2} h_{\mu \nu}-\partial_{\mu} \partial_{\nu} h\right), \\
& \partial^{2} \equiv \eta^{\mu \nu} \partial_{\mu} \partial_{\nu}, \quad h \equiv h_{\mu \nu} \eta^{\mu \nu}
\end{aligned}
$$

Eq. (9) becomes the ordinary energy-momentum conservation law

$$
\partial_{\mu} T^{\mu \nu}=0
$$

Since all tensor quantities under consideration $\left(T^{\mu \nu}, h_{\mu \nu}\right.$ etc.) are small, their indices are raised and lowered with the help of the flat-space metric $\eta_{\mu \nu}, \eta^{\mu \nu}$. Remembering the form of generators of the gauge transformations Eq. (14), we see that because of the conservation law Eq. (36), all diagrams of the type shown in Fig. 1(f) are equal to zero.

The ordinary Slavnov identities also help us to show that the sum of diagrams in Figs. 2(c) and 2(d) is equal to the diagram pictured in Fig. 1(d) For this purpose, we neglect device contribution, differentiate Eq. (31) twice with respect to $\beta_{\alpha}$ and $T^{\mu \nu}$, and set all the sources, including $T$, equal to zero

$$
\frac{\delta^{2} W}{\delta \beta_{\alpha} \delta K^{\mu \nu}}-\frac{1}{\xi} F^{\alpha, \sigma \lambda} \frac{\delta^{2} W}{\delta T^{\sigma \lambda} \delta T^{\mu \nu}}=0 .
$$

This is the well-known first Slavnov identity from which, in particular, the absence of radiation corrections to the longitudinal part of the graviton propagator follows. At the tree level, it reads

$$
\begin{aligned}
& \frac{1}{\xi} F^{\alpha, \mu \nu} G_{\mu \nu \sigma \lambda}(x)=-D_{\sigma \lambda}^{(0) \beta} \tilde{G}_{\beta}^{\alpha}(x), \\
& D_{\mu \nu}^{(0) \alpha} \equiv D_{\mu \nu}^{\alpha}(h=0),
\end{aligned}
$$

and is easily verified with the help of explicit expressions for the graviton and ghost propagators, $G_{\mu \nu \sigma \lambda}, \tilde{G}_{\alpha}^{\beta}$, given below [see Eqs. (40), (41)]. At the one-loop approximation, identity (37), contracted with $T^{\mu \nu}$, is shown in Fig. 5 .

This figure, together with the identity (38) and the relation

$$
\frac{\delta S_{\phi}}{\delta h_{\mu \nu}} D_{\mu \nu}^{\alpha} C_{\alpha}=-\frac{\delta S_{\phi}}{\delta \phi} \tilde{D}^{\alpha} C_{\alpha}
$$




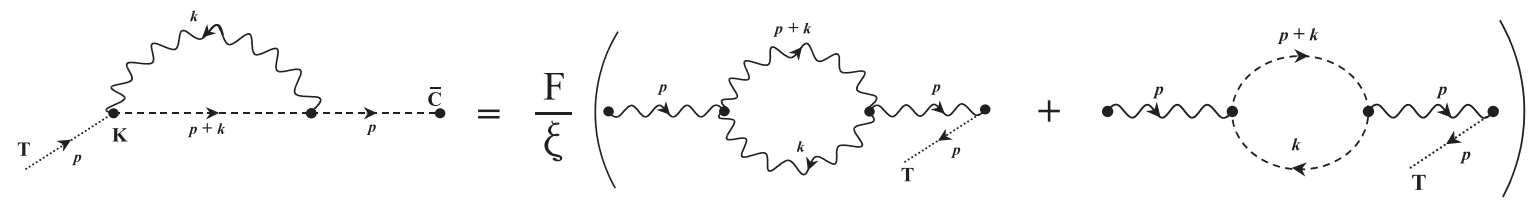

Figure 5: Graphical representation of the one-loop Slavnov identity (37) contracted with $T^{\mu \nu}$.

makes it clear that the sum of diagrams in Figs. 2(c,d) is equal to that pictured in Fig. 11(d). We would like to emphasize that the diagrams 2(c,d) are not equal to zero, as would be the case if the background field method were used.

Finally, diagrams 11(e) and 3(c) are zeros identically. As for the latter, this follows simply from the fact that there is no external momentum flow in the dimensionallyregularized loop integral, while in the former we have 0/0-type indefiniteness. It is easy to see, however, that this tadpole is to be set zero. Indeed, let the constant anticommuting source $Y$ be considered as the limit of a sequence of functions with infinitely expanding carriers on which the functions have the same constant value except for immediate neighborhood of the boundaries where they fall off to zero. Then the above indefiniteness turns into $q^{3} / q^{2}$, where $q$ is the tending to zero momentum flow through the $Y$-vertex.

Thus, the diagrams of Figs. 1 $(a, b, c, d), 2(a, b)$, and 3(a,b) remain to be calculated.

Since each diagram contains only one $\phi$-vertex, two sets of diagrams corresponding to the two terms in Eq. (13) must cancel independently. Below we present detailed calculation of these diagrams with $\phi$-vertices generated by the mass term. Evidently, one has to expand this term up to the second order in the gravitational field

$$
-\frac{1}{2} m^{2} \phi^{2} \sqrt{-g}=-\frac{1}{2} m^{2} \phi^{2}\left(1+\frac{1}{2} h+\frac{1}{8} h^{2}-\frac{1}{4} h^{\alpha \beta} h_{\alpha \beta}+O\left(h^{3}\right)\right) .
$$

We also calculate:

the second variation of Einstein action (11)

$\left.\frac{\delta^{2} S}{\delta h_{\sigma \lambda}(x) \delta h_{\mu \nu}(y)}\right|_{h=0}=\left\{-\frac{1}{4}\left(\eta^{\mu \sigma} \eta^{\nu \lambda}+\eta^{\mu \lambda} \eta^{\nu \sigma}-2 \eta^{\mu \nu} \eta^{\sigma \lambda}\right) \partial^{2}-\frac{1}{2}\left(\eta^{\sigma \lambda} \partial^{\mu} \partial^{\nu}+\eta^{\mu \nu} \partial^{\sigma} \partial^{\lambda}\right)\right.$
$\left.+\frac{1}{4}\left(\eta^{\sigma \mu} \partial^{\lambda} \partial^{\nu}+\eta^{\lambda \mu} \partial^{\sigma} \partial^{\nu}+\eta^{\sigma \nu} \partial^{\lambda} \partial^{\mu}+\eta^{\lambda \nu} \partial^{\sigma} \partial^{\mu}\right)\right\} \delta(x-y), \quad \partial^{2} \equiv \eta^{\mu \nu} \partial_{\mu} \partial_{\nu}, \quad \partial_{\mu} \equiv \frac{\partial}{\partial x^{\mu}}$

the graviton propagator $G_{\mu \nu \sigma \lambda}$ defined by

$$
\frac{\delta^{2} S}{\delta h_{\rho \tau} \delta h_{\mu \nu}} G_{\mu \nu \sigma \lambda}=-\delta_{\sigma \lambda}^{\rho \tau},
$$




$$
\begin{aligned}
& G_{\mu \nu \sigma \lambda}=\left(\eta_{\mu \sigma} \eta_{\nu \lambda}+\eta_{\mu \lambda} \eta_{\nu \sigma}-\eta_{\mu \nu} \eta_{\sigma \lambda}\right) \frac{1}{\partial^{2}}+2(\alpha+1)\left(\eta_{\mu \nu} \partial_{\sigma} \partial_{\lambda}+\eta_{\sigma \lambda} \partial_{\mu} \partial_{\nu}\right) \frac{1}{\partial^{4}} \\
& +(\xi-1)\left(\eta_{\mu \sigma} \partial_{\nu} \partial_{\lambda}+\eta_{\mu \lambda} \partial_{\nu} \partial_{\sigma}+\eta_{\nu \sigma} \partial_{\mu} \partial_{\lambda}+\eta_{\nu \lambda} \partial_{\mu} \partial_{\sigma}\right) \frac{1}{\partial^{4}} \\
& +\left(4 \alpha^{2} \xi-12 \alpha^{2}-16 \alpha-4 \xi-4\right) \partial_{\mu} \partial_{\nu} \partial_{\sigma} \partial_{\lambda} \frac{1}{\partial^{6}}, \quad \alpha \equiv \frac{1}{\beta-1},
\end{aligned}
$$

and the ghost propagator

$$
\tilde{G}_{\beta}^{\alpha}=-\frac{\delta_{\beta}^{\alpha}}{\partial^{2}}-\frac{\beta}{1-\beta} \frac{\partial^{\alpha} \partial_{\beta}}{\partial^{4}}
$$

satisfying

$$
F_{\alpha}^{, \mu \nu} D_{\mu \nu}^{(0) \beta} \tilde{G}_{\beta}^{\gamma}=-\delta_{\alpha}^{\gamma} .
$$

The three-graviton vertex encountered in diagrams 1(c), 2(a), and 3(b), need not be calculated explicitly. Indeed, with the help of the identity (38) the graviton propagator entering the vertex $Y$ can be substituted by the ghost propagator, the corresponding generator $D_{\mu \nu}^{(0) \alpha}$ being attached to the three-graviton vertex. The latter, therefore, can be expressed through the second variation of Einstein action

$$
\left.\frac{\delta^{3} S}{\delta h_{\mu \nu} \delta h_{\sigma \lambda} \delta h_{\rho \tau}}\right|_{h=0} D_{\mu \nu}^{(0) \alpha}+\left.\frac{\delta^{2} S}{\delta h_{\mu \nu} \delta h_{\rho \tau}}\right|_{h=0} \frac{\delta D_{\mu \nu}^{\alpha}}{\delta h_{\sigma \lambda}}+\left.\frac{\delta^{2} S}{\delta h_{\mu \nu} \delta h_{\sigma \lambda}}\right|_{h=0} \frac{\delta D_{\mu \nu}^{\alpha}}{\delta h_{\rho \tau}}=0 .
$$

This is obtained by double differentiating the basic identity

$$
\frac{\delta S}{\delta h_{\mu \nu}} D_{\mu \nu}^{\alpha}=0 .
$$

Thus, diagrams to be calculated take the following analytical form

$$
\begin{aligned}
& 1 a=-i\left\{-\frac{1}{2} m^{2} \phi^{2}(-p)\right\} E^{\mu \nu}(p) \mu^{\varepsilon} \int \frac{d^{4-\varepsilon} k}{(2 \pi)^{4}}\left(\frac{1}{4} \eta^{\tau \rho} \eta^{\sigma \lambda}-\frac{1}{2} \delta^{\tau \rho, \sigma \lambda}\right) \\
& \times G_{\tau \rho \chi \theta}(k)\left\{k_{\alpha} \delta_{\mu \nu}^{\chi \theta}-\delta_{\mu \alpha}^{\chi \theta}\left(k_{\nu}+p_{\nu}\right)-\delta_{\nu \alpha}^{\chi \theta}\left(k_{\mu}+p_{\mu}\right)\right\} \\
& \times \tilde{G}_{\beta}^{\alpha}(p+k) \xi \tilde{G}^{\beta \gamma}(p+k)\left\{\eta_{\sigma \gamma}\left(k_{\lambda}+p_{\lambda}\right)+\eta_{\lambda \gamma}\left(k_{\sigma}+p_{\sigma}\right)\right\}, \\
& 1 b=-i\left\{-\frac{1}{2} m^{2} \phi^{2}(-p)\right\} E^{\mu \nu}(p) \mu^{\varepsilon} \int \frac{d^{4-\varepsilon} k}{(2 \pi)^{4}} \tilde{G}_{\beta}^{\alpha}(p+k) \\
& \times\left\{\frac{1+\beta}{2}\left(p^{\beta}+k^{\beta}\right) \eta^{\tau \rho}-\frac{1}{2}\left(p^{\tau}+k^{\tau}\right) \eta^{\beta \rho}-\frac{1}{2}\left(p^{\rho}+k^{\rho}\right) \eta^{\beta \tau}\right\} \\
& \quad \times\left\{-p_{\gamma} \delta_{\tau \rho}^{\chi \theta}-\delta_{\tau \gamma}^{\chi \theta} k_{\rho}-\delta_{\rho \gamma}^{\chi \theta} k_{\tau}\right\} G_{\chi \theta \varphi \psi}(p) \frac{\eta^{\varphi \psi}}{2} \tilde{G}_{\delta}^{\gamma}(k) \xi \tilde{G}^{\delta \zeta}(k) \\
& \quad \times\left\{\eta_{\sigma \zeta} k_{\lambda}+\eta_{\lambda \zeta} k_{\sigma}\right\}\left\{k_{\alpha} \delta_{\mu \nu}^{\sigma \lambda}-\delta_{\mu \alpha}^{\sigma \lambda}\left(k_{\nu}+p_{\nu}\right)-\delta_{\nu \alpha}^{\sigma \lambda}\left(k_{\mu}+p_{\mu}\right)\right\}, \\
& 1 c=-i\left\{-\frac{1}{2} m^{2} \phi^{2}(-p)\right\} E^{\mu \nu}(p) \mu^{\varepsilon} \int \frac{d^{4-\varepsilon} k}{(2 \pi)^{4}} \tilde{G}_{\beta}^{\alpha}(p+k) \xi \tilde{G}^{\beta \gamma}(p+k) \\
& \times\left(-S^{, \sigma \lambda} \tau \rho(p)\left\{-k_{\gamma} \delta_{\sigma \lambda}^{\chi \theta}+\delta_{\sigma \gamma}^{\chi \theta}\left(k_{\lambda}+p_{\lambda}\right)+\delta_{\lambda \gamma}^{\chi \theta}\left(k_{\sigma}+p_{\sigma}\right)\right\}\right. \\
& \left.-S^{, \sigma \lambda} \chi \theta(k)\left\{-p_{\gamma} \delta_{\sigma \lambda}^{\tau \rho}+\delta_{\sigma \gamma}^{\tau \rho}\left(k_{\lambda}+p_{\lambda}\right)+\delta_{\lambda \gamma}^{\tau \rho}\left(k_{\sigma}+p_{\sigma}\right)\right\}\right) \\
& \times G_{\tau \rho \varphi \psi}(k) G_{\chi \theta \kappa \omega}(p) \frac{\eta^{\kappa \omega}}{2}\left\{k_{\alpha} \delta_{\mu \nu}^{\varphi \psi}-\delta_{\mu \alpha}^{\varphi \psi}\left(k_{\nu}+p_{\nu}\right)-\delta_{\nu \alpha}^{\varphi \psi}\left(k_{\mu}+p_{\mu}\right)\right\},
\end{aligned}
$$




$$
\begin{aligned}
& 1 d=-i\left\{-\frac{1}{2} m^{2} \phi^{2}(-p)\right\} E^{\mu \nu}(p) \mu^{\varepsilon} \int \frac{d^{4-\varepsilon} k}{(2 \pi)^{4}} G_{\kappa \omega \chi}(k) \\
& \times\left\{k_{\alpha} \delta_{\mu \nu}^{\chi \theta}-\delta_{\mu \alpha}^{\chi \theta}\left(k_{\nu}+p_{\nu}\right)-\delta_{\nu \alpha}^{\chi \theta}\left(k_{\mu}+p_{\mu}\right)\right\} \tilde{G}_{\beta}^{\alpha}(p+k) \\
& \times\left\{\frac{1+\beta}{2}\left(p^{\beta}+k^{\beta}\right) \eta^{\tau \rho}-\frac{1}{2}\left(p^{\tau}+k^{\tau}\right) \eta^{\beta \rho}-\frac{1}{2}\left(p^{\rho}+k^{\rho}\right) \eta^{\beta \tau}\right\} \\
& \times\left\{-k_{\gamma} \delta_{\tau \rho}^{\kappa \omega}-\delta_{\tau \gamma}^{\kappa \omega} p_{\rho}-\delta_{\rho \gamma}^{\kappa \omega} p_{\tau}\right\} \tilde{G}_{\delta}^{\gamma}(p) \xi \tilde{G}^{\delta \zeta}(p)\left\{\eta_{\sigma \zeta} p_{\lambda}+\eta_{\lambda \zeta} p_{\sigma}\right\} \frac{\eta^{\sigma \lambda}}{2} \\
& 2 a=-i\left\{-\frac{1}{2} m^{2} \phi^{2}(p)\right\} \mu^{\varepsilon} \int \frac{d^{4-\varepsilon} k}{(2 \pi)^{4}} \tilde{G}_{\beta}^{\alpha}(p+k) \xi \tilde{G}^{\beta \gamma}(p+k) \\
& \times\left(-S^{, \sigma \lambda \tau \rho}(p)\left\{-k_{\gamma} \delta_{\sigma \lambda}^{\chi \theta}+\delta_{\sigma \gamma}^{\chi \theta}\left(k_{\lambda}+p_{\lambda}\right)+\delta_{\lambda \gamma}^{\chi \theta}\left(k_{\sigma}+p_{\sigma}\right)\right\}\right. \\
& \left.-S^{, \sigma \lambda} \chi \theta(k)\left\{-p_{\gamma} \delta_{\sigma \lambda}^{\tau \rho}+\delta_{\sigma \gamma}^{\tau \rho}\left(k_{\lambda}+p_{\lambda}\right)+\delta_{\lambda \gamma}^{\tau \rho}\left(k_{\sigma}+p_{\sigma}\right)\right\}\right) \\
& \times h_{\chi \theta}(-p) G_{\tau \rho \varphi \psi}(k)\left\{\frac{1+\beta}{2} p^{\delta} \eta^{\mu \nu}-\frac{1}{2} p^{\mu} \eta^{\delta \nu}-\frac{1}{2} p^{\nu} \eta^{\delta \mu}\right\} \\
& \times\left\{k_{\alpha} \delta_{\mu \nu}^{\varphi \psi}-\delta_{\mu \alpha}^{\varphi \psi}\left(k_{\nu}+p_{\nu}\right)-\delta_{\nu \alpha}^{\varphi \psi}\left(k_{\mu}+p_{\mu}\right)\right\} \tilde{G}_{\delta}^{\zeta}(p) p_{\zeta}, \\
& 2 b=-i\left\{-\frac{1}{2} m^{2} \phi^{2}(p)\right\} \mu^{\varepsilon} \int \frac{d^{4-\varepsilon} k}{(2 \pi)^{4}} \tilde{G}_{\beta}^{\alpha}(p+k) \\
& \times\left\{\frac{1+\beta}{2}\left(p^{\beta}+k^{\beta}\right) \eta^{\tau \rho}-\frac{1}{2}\left(p^{\tau}+k^{\tau}\right) \eta^{\beta \rho}-\frac{1}{2}\left(p^{\rho}+k^{\rho}\right) \eta^{\beta \tau}\right\} \\
& \times\left\{-p_{\gamma} h_{\tau \rho}(-p)-h_{\tau \gamma}(-p) k_{\rho}-h_{\rho \gamma}(-p) k_{\tau}\right\} \tilde{G}_{\delta}^{\gamma}(k) \xi \tilde{G}^{\delta \zeta}(k) \\
& \times\left\{\eta_{\sigma \zeta} k_{\lambda}+\eta_{\lambda \zeta} k_{\sigma}\right\}\left\{k_{\alpha} \delta_{\mu \nu}^{\sigma \lambda}-\delta_{\mu \alpha}^{\sigma \lambda}\left(k_{\nu}+p_{\nu}\right)-\delta_{\nu \alpha}^{\sigma \lambda}\left(k_{\mu}+p_{\mu}\right)\right\} \\
& \times\left\{\frac{1+\beta}{2} p^{\epsilon} \eta^{\mu \nu}-\frac{1}{2} p^{\mu} \eta^{\epsilon \nu}-\frac{1}{2} p^{\nu} \eta^{\epsilon \mu}\right\} \tilde{G}_{\epsilon}^{\eta}(p) p_{\eta}, \\
& 3 a=-i\left\{-\frac{1}{2} m^{2} \phi^{2}(p)\right\} \mu^{\varepsilon} \int \frac{d^{4-\varepsilon} k}{(2 \pi)^{4}} \tilde{G}_{\beta}^{\alpha}(p+k) p_{\alpha}\left\{\eta_{\sigma \zeta} k_{\lambda}+\eta_{\lambda \zeta} k_{\sigma}\right\} \frac{\eta^{\sigma \lambda}}{2} \\
& \times\left\{\frac{1+\beta}{2}\left(p^{\beta}+k^{\beta}\right) \eta^{\tau \rho}-\frac{1}{2}\left(p^{\tau}+k^{\tau}\right) \eta^{\beta \rho}-\frac{1}{2}\left(p^{\rho}+k^{\rho}\right) \eta^{\beta \tau}\right\} \\
& \times\left\{-p_{\gamma} h_{\tau \rho}(-p)-h_{\tau \gamma}(-p) k_{\rho}-h_{\rho \gamma}(-p) k_{\tau}\right\} \tilde{G}_{\delta}^{\gamma}(k) \xi \tilde{G}^{\delta \zeta}(k), \\
& 3 b=-i\left\{-\frac{1}{2} m^{2} \phi^{2}(p)\right\} \mu^{\varepsilon} \int \frac{d^{4-\varepsilon} k}{(2 \pi)^{4}} p_{\alpha} \tilde{G}_{\beta}^{\alpha}(p+k) \xi \tilde{G}^{\beta \gamma}(p+k) \\
& \times\left(-S^{, \sigma \lambda \tau \rho}(p)\left\{-k_{\gamma} \delta_{\sigma \lambda}^{\chi \theta}+\delta_{\sigma \gamma}^{\chi \theta}\left(k_{\lambda}+p_{\lambda}\right)+\delta_{\lambda \gamma}^{\chi \theta}\left(k_{\sigma}+p_{\sigma}\right)\right\}\right. \\
& \left.-S^{, \sigma \lambda} \chi^{\theta}(k)\left\{-p_{\gamma} \delta_{\sigma \lambda}^{\tau \rho}+\delta_{\sigma \gamma}^{\tau \rho}\left(k_{\lambda}+p_{\lambda}\right)+\delta_{\lambda \gamma}^{\tau \rho}\left(k_{\sigma}+p_{\sigma}\right)\right\}\right) \\
& \times h_{\chi \theta}(-p) G_{\tau \rho \mu \nu}(k) \frac{\eta^{\mu \nu}}{2} \text {, }
\end{aligned}
$$


where $E^{\mu \nu}$ stands for the Einstein tensor

$$
E^{\mu \nu}=R^{\mu \nu}-\frac{1}{2} \eta^{\mu \nu} R_{\alpha \beta} \eta^{\alpha \beta},
$$

$\phi^{2}(p)$ - Fourier transform of the square of the scalar field, $\mu$ - arbitrary mass scale, and $\varepsilon=4-d, d$ being the dimensionality of space-time.

As we see, all Feynman integrals we need to calculate have the following form

$$
I(p)=\mu^{\varepsilon} \int d^{4-\varepsilon} k f(p, k),
$$

where $f(p, k)$ is the product of the graviton and ghost propagators and of the vertex factors. Since we neglect quantum propagation of the scalar field, there is no dimensional parameters in the integrands. Therefore, as a simple dimensional analysis shows, $I(p)$ have the following structure

$$
I=c_{1} p^{N}\left(\frac{\mu^{2}}{p^{2}}\right)^{\frac{\varepsilon}{2}}\left[\frac{1}{\varepsilon}+c_{2}\right]=c_{1} p^{N}\left[\frac{1}{\varepsilon}-\frac{1}{2} \ln \left(\frac{p^{2}}{\mu^{2}}\right)+c_{2}+O(\varepsilon)\right],
$$

$c_{1}, c_{2}$ and $N$ being some numbers depending on the specific form of $f(p, k)$. It follows from Eq. (45) that one can obtain the logarithmic contribution from divergent one substituting

$$
\frac{1}{\varepsilon} \rightarrow-\frac{1}{2} \ln \left(\frac{p^{2}}{\mu^{2}}\right)
$$

All the above Feynman integrals are ultraviolet divergent. It is important, on the other hand, that they are free of infrared divergences. Indeed, denominators in these integrals are the products of only two scalars $-(p+k)^{2}$ and $k^{2}$. If we rewrite also every $p$ entering the vertex factors as $(p+k)-k$, then the integrands take the form of sums of products of powers $(p+k)^{n}$ and $k^{m}$. Since all diagrams are ultraviolet divergent, we have $n+m \geq-4$. On the other hand, infrared divergences appear only if $n \leq-4$, or $m \leq-4$, and, therefore, we have $m \geq 0$, or $n \geq 0$, respectively. In either case the dimensionally regularized loop integrals turn into zero.

Thus, to calculate the logarithmic contribution of the diagrams it is sufficient to find their ultraviolet divergences. For this purpose, we expand all denominators in a finite series in powers of $p / k$ keeping only first $N$ terms. It is convenient to apply identity (38) to the $Y$-vertex in all diagrams under consideration, since then such an expansion is to be performed on the ghost propagator only

$$
\begin{aligned}
& \tilde{G}_{\beta}^{\alpha}(p+k)=-\frac{\delta_{\beta}^{\alpha}}{k^{2}}\left(1-\frac{2(p k)}{k^{2}}-\frac{p^{2}}{k^{2}}+\frac{4(p k)^{2}}{k^{4}}+\frac{4(p k) p^{2}}{k^{4}}-\frac{8(p k)^{3}}{k^{6}}\right) \\
& -\frac{\beta}{1-\beta} \frac{(k+p)^{\alpha}(k+p)_{\beta}}{k^{4}}\left(1-\frac{4(p k)}{k^{2}}-\frac{2 p^{2}}{k^{2}}+\frac{12(p k)^{2}}{k^{4}}+\frac{12(p k) p^{2}}{k^{4}}-\frac{32(p k)^{3}}{k^{6}}\right) \\
& +O\left(\frac{1}{k^{6}}\right) .
\end{aligned}
$$

Now, calculation of diagrams is straightforward. The tensor multiplication as well as integration over angles in the momentum space is performed with the help of the New Tensor Package for REDUCE System [18]. The result of the calculation is the following. Making use of the gauge condition (12), one can reduce the functional structure of each diagram to $p^{2} h(-p) \phi^{2}(p)$, the coefficients being polynomials on the

\footnotetext{
${ }^{9}$ The subscripts of the polynomials refer to the corresponding diagrams of Figs. 1 t 3 .
} 
gauge parameters $\alpha \equiv(\beta-1)^{-1}$ and $\xi$

$$
I(p)=\left\{-\frac{1}{2} m^{2} p^{2} h(-p) \phi^{2}(p)\right\}\left(\frac{\mu^{2}}{p^{2}}\right)^{\varepsilon / 2} \frac{P(\alpha, \xi)}{8 \pi^{2} \varepsilon},
$$

where

$$
\begin{gathered}
P_{1 a}(\alpha, \xi)=-\frac{1}{4} \alpha^{3} \xi^{2}+\frac{3}{4} \alpha^{3} \xi+\frac{3}{2} \alpha^{2} \xi+\frac{3}{4} \alpha \xi-\frac{3}{4} \alpha^{-1} \xi^{2} \\
P_{1 b}(\alpha, \xi)=-\frac{5}{32} \alpha^{4} \xi^{2}+\frac{15}{32} \alpha^{4} \xi-\frac{3}{32} \alpha^{3} \xi^{2}+\frac{29}{96} \alpha^{3} \xi \\
+\frac{35}{96} \alpha^{2} \xi^{2}-\frac{103}{96} \alpha^{2} \xi+\frac{15}{32} \alpha \xi^{2}-\frac{75}{32} \alpha \xi-\frac{7}{16} \xi \\
P_{1 c}(\alpha, \xi)=-\frac{55}{96} \alpha^{4} \xi^{2}+\frac{55}{32} \alpha^{4} \xi-\frac{3}{32} \alpha^{3} \xi^{2}+\frac{253}{96} \alpha^{3} \xi \\
+\frac{35}{96} \alpha^{2} \xi^{2}-\frac{19}{96} \alpha^{2} \xi+\frac{15}{32} \alpha \xi^{2}-\frac{75}{32} \alpha \xi-\frac{5}{4} \xi^{2}-\frac{17}{16} \xi \\
P_{1 d+2 c+2 d}(\alpha, \xi)=-\frac{5}{16} \alpha^{4} \xi^{2}+\frac{15}{16} \alpha^{4} \xi-\frac{3}{16} \alpha^{3} \xi^{2} \\
+\frac{125}{48} \alpha^{3} \xi+\frac{35}{48} \alpha^{2} \xi^{2}+\frac{127}{48} \alpha^{2} \xi+\frac{15}{16} \alpha \xi^{2}+\frac{31}{48} \alpha \xi \\
P_{2 b}(\alpha, \xi)=\frac{15}{32} \alpha^{4} \xi+\frac{41}{96} \alpha^{3} \xi-\frac{131}{96} \alpha^{2} \xi \\
P_{2 a}(\alpha, \xi)=-\frac{5}{12} \alpha^{4} \xi^{2}+\frac{55}{32} \alpha^{4} \xi-\frac{1}{4} \alpha^{3} \xi^{2}+\frac{361}{96} \alpha^{3} \xi \\
+\frac{161}{96} \alpha^{2} \xi-\frac{221}{96} \alpha \xi-\frac{3}{4} \alpha^{-1} \xi^{2}-\frac{7}{12} \alpha^{-1} \xi-\frac{5}{4} \xi^{2}-\frac{137}{48} \xi \\
P_{3 a}(\alpha, \xi)=\frac{1}{8} \alpha^{3} \xi+\frac{11}{24} \alpha^{2} \xi+\frac{1}{12} \alpha \xi \\
\hline
\end{gathered}
$$

Finally, summing up individual contributions, taking into account

$$
\square h=2 \alpha R=2 \alpha T, \quad T=T^{\mu \nu} \eta_{\mu \nu},
$$

and going over to coordinate space we have for the total gauge dependence of the effective mass term of the scalar field action

$$
\frac{d \Gamma_{\phi}}{d \xi}=\left\{-\int d^{4} x \frac{1}{2} m^{2} \phi^{2}(x) \ln \square T(x)\right\} \frac{P^{t o t}(\alpha, \xi)}{16 \pi^{2}},
$$


where

$$
\begin{aligned}
& P^{\text {tot }}(\alpha, \xi)=\left(\frac{35}{24} \alpha^{5} \xi-\frac{85}{16} \alpha^{5}+\frac{7}{8} \alpha^{4} \xi-\frac{503}{48} \alpha^{4}-\frac{35}{24} \alpha^{3} \xi\right. \\
& \left.-\frac{55}{16} \alpha^{3}-\frac{15}{8} \alpha^{2} \xi+\frac{475}{48} \alpha^{2}+\frac{5}{2} \alpha \xi+\frac{23}{3} \alpha+\frac{3}{2} \xi+\frac{7}{6}\right)
\end{aligned}
$$

Although we have only determined gauge dependence of the effective mass term, this is enough to conclude that the effective equations of motion of the apparatus depend on the choice of the gauge. One might think that these equations could still turn out to be gauge-independent provided that the effective kinetic term had an appropriate gaugedependent part, e.g., the one described by equation analogous to Eq. (48) with the same polynomial $P^{\text {tot }}(\alpha, \xi)$. Remember, however, that besides the terms of the order $\hbar^{1}$, whose $\xi$-dependence is described by Eq. (48), the effective apparatus action contains also terms of the order $\hbar^{0}$, corresponding to the classical (tree) approximation. The latter are of course gauge-independent, so there can be no cancellation of the gaugedependent factors in the effective equations of motion. Obviously, no manipulation with these equations can change this conclusion, since Eq. (48) alone is already sufficient to determine the gauge dependence of the effective gravitational field. Indeed, as explained in the Introduction, this field is to be determined by comparison of the effective and classical equations of motion of the measuring device (or by comparison of the corresponding action functionals). The mass term in the action (13) for the scalar particle in a weak gravitational field is

$$
-\int d^{4} x \frac{1}{2} m^{2} \phi^{2}(x) \frac{h_{\mu \nu}(x) \eta^{\mu \nu}}{2}
$$

Therefore, in view of arbitrariness of the source $T^{\mu \nu}$, we conclude that the gauge dependence of the effective gravitational field measured by means of the scalar particle is described by the following equation

$$
\frac{d h_{\mu \nu}^{e f f}(x)}{d \xi}=\ln \square T_{\mu \nu}(x) \frac{P^{t o t}(\alpha, \xi)}{8 \pi^{2}} .
$$

Thus, unlike the case of the point-like measuring apparatus, considered in [5], the value of the effective gravitational field measured by the scalar field turns out to be gaugedependent. However, the non-vanishing of the right hand side of Eq. (49) should not discourage, since as we have mentioned in the Introduction this result does not take into account gauge dependence of the radiation corrections to the classical form of the scalar field action (i.e., the gravitational form factors of the scalar particle), which in the case of the gravitational interaction do not disappear in the limit $p^{2} / m^{2} \rightarrow 0$.

\section{Conclusion}

The results of the calculation presented in this paper show that from the field-theoretical point of view, explicit inclusion of the measuring apparatus into mathematical framework of the quantum theory does not solve the problem of gauge dependence of observables, unlike the case when the effective field is measured by point particles, discussed in [5]. 
It must be emphasized, however, that we have obtained this result under assumption that the action for the measuring device can be taken in the ordinary classical form. Unlike all other fundamental interactions, in the case of gravity this choice of the action cannot be justified by the standard limiting arguments [9]. In other words, consistent field-theoretical approach requires taking into account quantum corrections to the classical form of the device action, i.e., evaluation of the corresponding form factors. Such evaluation is by itself very complicated task, since one has to deal with Feynman graphs whose external lines all are off the mass-shell. Its results would be decisive for answering the question of whether approach suggested in [5] does solve the gauge dependence problem.

Leaving this question open we would like to note only that its eventual resolution would serve as a valuable guide in investigation of the fundamental role played by the measurement process in quantum theory, of which description presented above is just a phenomenology.

\section{Appendix}

As we saw in Sec. đ, the effective Slavnov identities for the combined system "gravitational field plus measuring device" contain the second derivatives of the generating functionals of Green functions with respect to the sources. If the apparatus contribution to the effective gravitational field is infinitesimal, this fact is inessential since then evaluation of the gauge dependence of the generating functional of simply connected Green functions is needed only [see Eq. (8)]. However, in the general case of finite device contribution, one has to deal with the effective Slavnov identity for the generating functional of one-particle-irreducible Green functions, i.e., effective action. In this case, one can avoid the complications caused by the presence of the second derivatives, which arise when one performs the Legendre transformation in Eq. (31). This can be done as follows.

As seen from the derivation of identities (30), (31), given in Sec. 4, appearance of the second derivatives in these identities is traced to non-invariance of the apparatus action with respect to the "quantum" BRST transformation (18) of integration variables in the generating functional (21). The first term in the square brackets in Eq. (27), representing this non-invariance, is non-linear on the quantum fields. On the other hand, the standard source for the BRST transformation of the field $\phi$ (the Zinn-Justin source, [16]) is linear on the quantum fields, since the generator $\tilde{D}(\phi)$ is a functional of the classical field $\phi$ only. In this sense, introduction of the source $\tilde{K}$ into the quantum action is superfluous. It is natural, therefore, to introduce a source for the BRST variation of the whole device action, instead of the source $\tilde{K}$. Namely, let us consider the generating functional (21), where $\Sigma$ stands for

$$
\Sigma=S_{f p}+K^{\mu \nu} D_{\mu \nu}^{\alpha} C_{\alpha}+\frac{1}{2} L^{\gamma} f_{\gamma}^{\alpha \beta} C_{\alpha} C_{\beta}+J \frac{\delta S_{\phi}}{\delta \phi_{i}} \tilde{D}_{i}^{\alpha} C_{\alpha}
$$

$J$ being the new constant anticommuting parameter. Here we consider the general case when the measuring device is described by an arbitrary number of fields $\phi_{i}$, denoting them collectively as $\phi$. Obviously, the new source term is invariant under the "quantum" BRST transformation (18). 
To derive the effective Slavnov identities, we do as in the beginning of Sec. 4 and obtain the following identity

$$
\begin{aligned}
& \int d h d C d \bar{C}\left[i \frac{\delta S_{\phi}}{\delta h_{\mu \nu}} D_{\mu \nu}^{\alpha} C_{\alpha}+i Y \bar{C}^{\alpha} F_{\alpha}^{, \mu \nu} D_{\mu \nu}^{\beta} C_{\beta}+i \frac{Y}{\xi} F_{\alpha}^{2}+T^{\mu \nu} \frac{\delta}{\delta K^{\mu \nu}}\right. \\
& \left.-\bar{\beta}^{\alpha} \frac{\delta}{\delta L^{\alpha}}-i \beta_{\alpha} \frac{F^{\alpha}}{\xi}\right] \exp \left\{i\left(\Sigma+Y F_{\alpha} \bar{C}^{\alpha}+\bar{\beta}^{\alpha} C_{\alpha}+\bar{C}^{\alpha} \beta_{\alpha}+T^{\mu \nu} h_{\mu \nu}\right)\right\}=0 .
\end{aligned}
$$

The first term in the square brackets is now replaced simply by the minus derivative with respect to the source $J$. Applying the ghost equation of motion to the second term, we rewrite Eq. (50) as

$$
\left(T^{\mu \nu} \frac{\delta}{\delta K^{\mu \nu}}-\bar{\beta}^{\alpha} \frac{\delta}{\delta L^{\alpha}}-\frac{1}{\xi} \beta_{\alpha} F^{\alpha, \mu \nu} \frac{\delta}{\delta T^{\mu \nu}}-\frac{\partial}{\partial J}-Y \beta_{\gamma} \frac{\delta}{\delta \beta_{\gamma}}-2 Y \xi \frac{\partial}{\partial \xi}\right) Z=0
$$

which in terms of the generating functional of connected Green functions takes the form

$$
T^{\mu \nu} \frac{\delta \tilde{W}}{\delta K^{\mu \nu}}-\bar{\beta}^{\alpha} \frac{\delta \tilde{W}}{\delta L^{\alpha}}-\frac{1}{\xi} \beta_{\alpha} F^{\alpha, \mu \nu} \frac{\delta \tilde{W}}{\delta T^{\mu \nu}}-\frac{\partial \tilde{W}}{\partial J}-Y \beta_{\alpha} \frac{\delta \tilde{W}}{\delta \beta_{\alpha}}-2 Y \xi \frac{\partial \tilde{W}}{\partial \xi}=0 .
$$

Now, the Legendre transformation is easily performed in Eq. (51): with the help of equations

$$
T^{\mu \nu}=-\frac{\delta \tilde{\Gamma}}{\delta h_{\mu \nu}}, \quad \bar{\beta}^{\alpha}=\frac{\delta \tilde{\Gamma}}{\delta C_{\alpha}}, \quad \beta_{\alpha}=-\frac{\delta \tilde{\Gamma}}{\delta \bar{C}^{\alpha}},
$$

which are the inverse of Eqs. (23) - (25), and the relations

$$
\frac{\delta \tilde{W}}{\delta K^{\mu \nu}}=\frac{\delta \tilde{\Gamma}}{\delta K^{\mu \nu}}, \quad \frac{\delta \tilde{W}}{\delta \xi}=\frac{\delta \tilde{\Gamma}}{\delta \xi}, \text { etc. }
$$

we bring Eq. (51) to

$$
\frac{\delta \tilde{\Gamma}}{\delta h_{\mu \nu}} \frac{\delta \tilde{\Gamma}}{\delta K^{\mu \nu}}+\frac{\delta \tilde{\Gamma}}{\delta C_{\alpha}} \frac{\delta \tilde{\Gamma}}{\delta L^{\alpha}}-\frac{F^{\alpha}}{\xi} \frac{\delta \tilde{\Gamma}}{\delta \bar{C}^{\alpha}}+\frac{\partial \tilde{\Gamma}}{\partial J}+Y \frac{\delta \tilde{\Gamma}}{\delta \bar{C}^{\alpha}} \bar{C}^{\alpha}+2 Y \xi \frac{\partial \tilde{\Gamma}}{\partial \xi}=0 .
$$

This is desired effective Slavnov identity for the generating functional of one-particleirreducible Green functions. Also, it is easy to verify that upon extraction of the

$\phi$-dependent terms in Eq. (51), the (one-loop) functional $\partial \tilde{W} / \partial J$ generates just the set of diagrams in Figs. 2, 3.

\section{References}

[1] J. Goldstone, A. Salam, and S. Weinberg, Phys. Rev. 127, 965 (1962).

[2] G. Jona-Lasinio, Nuovo Cimento 34, 1790 (1964).

[3] K. A. Kazakov and P. I. Pronin, Phys. Rev. D 59, 064012 (1999); K. A. Kazakov, P. I. Pronin, and K. V. Stepanyantz, Grav. Cosmol. 1, 17 (1998). 
[4] W. Heisenberg, in Niels Bohr and the Development of Physics (Pergammon Press, London, 1955).

[5] D. Dalvit and F. Mazzitelli, Phys.Rev. D 56, 7779 (1997); Quantum corrections to the geodesic equation, Talk presented at the meeting Trends in Theoretical Physics II, Buenos Aires, Argentina (1998).

[6] K. A. Kazakov and P. I. Pronin, Nucl. Phys. B573, 536 (2000).

[7] C. Becchi, A. Rouet, and R. Stora, Ann. of Phys. 98, 287 (1976); Commun. Math. Phys. 42, 127 (1975); I. V. Tyutin, Report FIAN 39 (1975).

[8] L. D. Faddeev and V. N. Popov, Phys. Lett. 25B, 29 (1967).

[9] J. F. Donoghue, Phys.Rev. D 50, 3874 (1994).

[10] B. S. DeWitt, Phys. Rev. 162, 1195 (1967);

[11] G.'t Hooft, in: Acta Universitatus Wratislavensis N368, XIIth Winter School of Theoretical Physics in Karpacz, February-March, 1975. Functional and Probabilistic Methods in Quantum Field Theory, vol.I; B. S. DeWitt, in: Quantum Gravity 2, edited by C. J. Isham, R. Penrose, and D. W. Sciama, (Clarendon Press, Oxford, 1981); D. G. Boulware, Phys. Rev. D 23, 389 (1981); L. F. Abbott, Nucl. Phys. B185, 189 (1981); C. F. Hart, Phys. Rev. D 28, 1993 (1983).

[12] I. A. Batalin and G. A. Vilkovisky, Phys. Lett. 102B, (1981), 27.

[13] P. M. Lavrov, I. V. Tyutin, and B. L. Voronov, Yad. Fiz. v.36, 498 (1982) [Sov. J. Nucl. Phys. 36, 292 (1982)].

[14] R. Kallosh and I. V. Tyutin, Yad. Fiz. v.17, 190 (1973) [Sov. J. Nucl. Phys. 17, $98(1973)]$.

[15] G. A. Vilkovisky, in Quantum Theory of Gravity, edited by S. M. Christensen (Hilger, Bristol, 1984), p. 169; Nucl. Phys. B234, 125 (1984).

[16] J. Zinn-Justin, in Trends in Elementary Particle Physics, edited by H. Rollnik and K. Dietz (Springer-Verlag, Berlin, 1975), p. 2.

[17] N. K. Nielsen, Nucl. Phys. B101, 173 (1975); H. Kluberg-Stern and J. B. Zuber, Phys. Rev. D 12, 467, 3159 (1975).

[18] P. I. Pronin and K. V. Stepanyantz, in New Computing Technick in Physics Research. IV., edited by B. Denby and D. Perred-Gallix (World Scientific, Singapure, 1995), p. 187. 